\title{
EQUIVALENT STRESS IN A PRESSURE VESSEL HEAD WITH A NOZZLE
}

\author{
JERZY LEWIŃSKI \\ Poznan University of Technology, Institute of Applied Mechanics, Poznań, Poland \\ e-mail: jerzy.lewinski@put.poznan.pl
}

\begin{abstract}
The paper is devoted to analysis of a dished head of a pressure vessel subject to internal uniform pressure and provided with a nozzle. A short survey of optimal design of the pressure vessel and its head is presented. The vessel head of a selected optimized shape is then completed by a nozzle, and the equivalent stress pattern is observed and recorded. The axis of symmetry of the nozzle is parallel to that of the vessel. The nozzle is placed in various distances from the axis of symmetry of the vessel. The dimensionless distance $e$ between both axes varies from 0 (i.e. axisymmetric location) up to 0.8 (assuming that the radius of cylindrical part of the vessel is equal to 1). Results of numerical calculation are presented in plots and figures.
\end{abstract}

Keywords: pressure vessel, dished head, nozzle-vessel strength

\section{Introduction}

The problem of dished heads of vessels has been undertaken by many investigators. Magnucki and Lewiński (2000) described the stress state arising in a untypical torispherical head composed of circular and polynomial parts. Magnucki and Lewiński (2003) presented the optimal design of an ellipsoidal head with consideration of various thickness values of the shell. Magnucki et al. (2004) presented optimization of a ground-based cylindrical tank with consideration of its strength and stability.

Błachut and Magnucki (2008) delivered a review of strength, static stability, and structural optimization of horizontal pressure vessels. Wittenbeck and Magnucki (2008) shaped the dished head generatrix in form of clothoidal and circular parts. Ventsel and Krauthammer (2001) delivered a monograph presenting the strength and stability problems of plates and shells with the edge effect of cylindrical shells. Magnucki et al. (2002) solved the problem of minimization of the stress concentration in a vessel provided with an ellipsoidal head. They considered various head-to-vessel thickness ratios and various values of relative convexity of the heads.

Lewiński and Magnucki (2010) considered a dished head of a pressure vessel subject to internal uniform pressure. They used a trigonometric series in order to ensure a proper shape of the profile. A similar approach to the shape of a dished head of a pressure vessel but with the use of the Bézier curves was presented by the same authors (2012). Further development of the method was demonstrated by Kruzelecki and Proszowski (2012). They considered a larger range of the shapes of a vessel head approximated by the convex Bézier polynomial or by functions with free parameters. The optimal solutions were obtained with the use of the simulated annealing algorithm. Szybiński and Wróblewski (2011) presented an interesting optimization of a stress relieving groove located between the vessel and a plate end closing it. The authors optimized the groove profile using two spline functions that resulted in reduction of the stress concentration.

Carbonari et al. (2011) presented integrated optimization of the shape of an axisymmetric vessel provided with a nozzle. They minimized the Huber-Mises mechanical stress in the whole vessel and, particularly, in the nozzle/vessel junction. 
Zhou et al. (1995) presented an analytical study on flexibility of nozzles in pressure vessel heads subject to radial thrust load. They used the shell theory and considered the effect of transverse shear deformation. Liu et al. (2001) sought optimal shapes of intersecting pressure vessels. They considered the optimal profile of a variable thickness nozzle connecting a spherical shell pressure vessel to a cylindrical nozzle. They showed that the design with the protruding nozzle gives a better stress distribution than a flush nozzle. Saal et al. (1997) considered the forces, moments, and stresses arising in the pressure vessel head and in the nozzle when the nozzle is located in the knuckle region of the vessel. They presented FEM analysis of the case and verified it by experimental investigation. Dekker and Brink (2000) analyzed the stress in the weld area of the nozzle/spherical vessel junction. They calculated the stress based on thin shell theory and verified the results with 3D-FEM. A similar problem was considered by Hsieh et al. (2000). The authors of this paper presented the limit loads for the case of internal pressure. Giglio (2003) compared two different methods of designing vessel nozzles, included in the ASME and VCR 1995 standards. He focused on the fatigue analysis of various pressure vessel nozzles. Skopinsky and Smetankin (2003) analyzed the stress arising in a reinforced nozzle/ellipsoidal head connection with the use of shell theory and the finite element method. Various reinforcement types were considered. Schindler and Zeman (2003) considered stress concentration factors for the case of the connection between the radial nozzle and spherical shell.

The present paper considers a dished head of a cylindrical pressure vessel subject to internal uniform pressure and provided with a cylindrical nozzle. The nozzle axis is parallel to the axis of the vessel. Several variants distinguished by various distances between both axes are taken into account.

\section{Formulation of the problem}

Vessels are usually provided with various holes, for example the ones designed as stack drains or serving as inlets or drain holes. According to specific purposes, the holes may be located in the cylindrical part of the vessel or in the head. The present paper considers a pressure vessel head provided with a nozzle designed for connecting the vessel to a pipeline. The nozzle is of circular cross section and a rather small length and diameter. Let us assume that the nozzle wall thickness is equal to that of the head. The paper is aimed at determining the equivalent stress distribution in the head in the vicinity of the nozzle as well as in the nozzle itself.

The shape of the head closing a cylindrical pressure vessel significantly affects the pattern of the stress arising in it. The shape of the vessel cross section is shown in Fig. 1. Radius of the cylindrical part of the vessel amounts to $a$, while the depth of the dished head is equal to $b$. Only a dimensionless profile of the vessel is here considered, hence, the radius of the cylindrical part of the vessel is equal to 1 , while the relative depth of the dished head amounts to $\beta=b / a$. The head is provided with a nozzle protruding to the length $L$ from the head (Fig. 1).

Since the stress depends, among others, on curvature of the vessel generatrix, the radius of the curvature should be continuous. Such a shape of the head generatrix may be described by a curve defined by the function $y(x)$ the coordinates $x(t), y(t)$ of which are expressed by the rational Bézier function. This allows one to alleviate considerably the stress concentration arising in the junction between the head and cylindrical part of the vessel.

According to the theory of momentless shells of revolution (Magnucki, 1998) and substituting the pressure, radius, and vessel wall thickness equal to unity, one obtains the equivalent Hubervon Mises stress in the cylindrical part of a thin-walled pressure vessel equal to $\widetilde{\sigma}_{e q}=\sqrt{3} / 2$. This value may be considered as a dimensionless stress. Analysis of such a dimensionless case enables more universal assessment of the stress state of the structure, instead of imposing fixed values of the size and load parameters. 
(a)

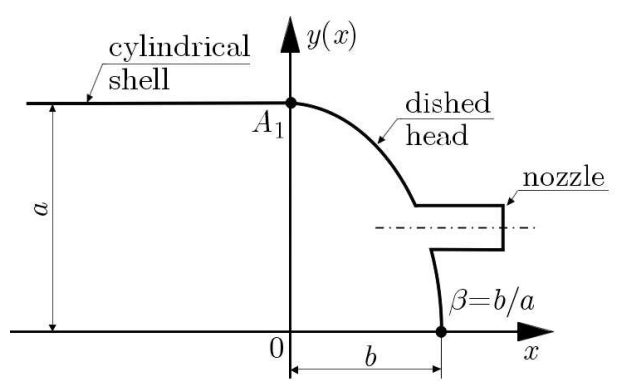

(b)

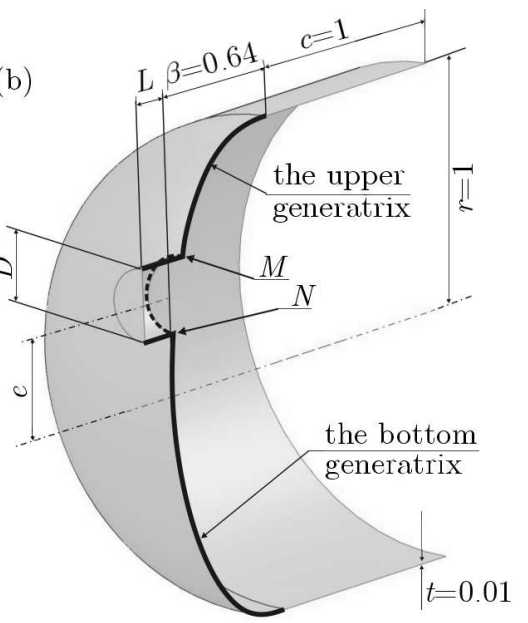

Fig. 1. A part of the vessel with the head and nozzle

An appropriate choice of the head profile enables reduction of the dimensionless head stress to the level not exceeding the stress existing in the cylindrical part of the vessel, i.e. to $\widetilde{\sigma}_{e q} \leqslant \sqrt{3} / 2$ in the case of the head without the nozzle (Lewiński and Magnucki, 2010).

Nevertheless, when the head is provided with a nozzle, the stress in the head/nozzle connection remains high and may be reduced with the help of other means. The zone of the head/nozzle connection is subject to disturbance of the membrane stress state existing in the vessel. In the case of symmetrical location of the nozzle, i.e. when the vessel axis and nozzle axis are identical, the stress distribution may be determined analytically (Magnucki, 1998). Otherwise, when both symmetry axes are shifted one with respect to another, the analytical approach would be extremely difficult.

The numerical analysis of equivalent stress in the head and nozzle is carried out for various positions of the nozzle with regard to the vessel: from symmetrical to eccentric locations of different eccentric shifts between the axes of symmetry.

The shape of the head is given by the Bézier function mentioned above. Dimensionless radius of the vessel is equal to $a=1$, with relative depth of the head amounting to $\beta=0.64$. It is identical with the one described by Lewiński and Magnucki (2012). The head is provided with a nozzle of the dimensionless diameter $D=0.3$, protruding to the distance $L=0.3$ beyond the head. The axis of symmetry of the nozzle is parallel to that of the cylindrical part of the vessel. It is located above the axis of symmetry of the vessel and shifted from it at the distance e (the eccentric shift). The parallel arrangement of both axes is here considered as in many hydraulic systems in which pipelines are located horizontally their connection to horizontally situated nozzles is more advantageous. Hence, the question arises whether the vessel head and the nozzle may be safely connected in such a case.

\section{Analysis of considered variants of the nozzle location}

The analysis is carried out with the help of an educational version of SolidWorks. The Simulation option of the software enables the finite element analysis of structures.

The dimensionless thickness of the shell in the examples calculated here is equal to $t=0.01$. The head is completed with a fragment of the cylindrical part of the vessel of the length $c=1$. Such a structure, when loaded with uniform internal pressure $p$, has a central vertical plane of symmetry. Therefore, the computational model shown in Fig. 1 includes only a half of the considered object. 
All the vessel cases calculated in the present paragraph are considered as thin-walled ones. In consequence, the vessel and nozzle walls are represented by their middle surfaces and are divided into triangular three-node shell elements. The numbers of nodes and elements in all the following cases amounted to about 60000 and 30000, respectively.

The analysis begins from the case for $e=0$, i.e. from a model of full axial symmetry. In this case, the upper and bottom generatrices (Fig. 1) are symmetrical, and the patterns of the stress along them are identical. The equivalent stress in this case is shown in Fig. 2. The stresses are plotted along the head and nozzle generatrices.

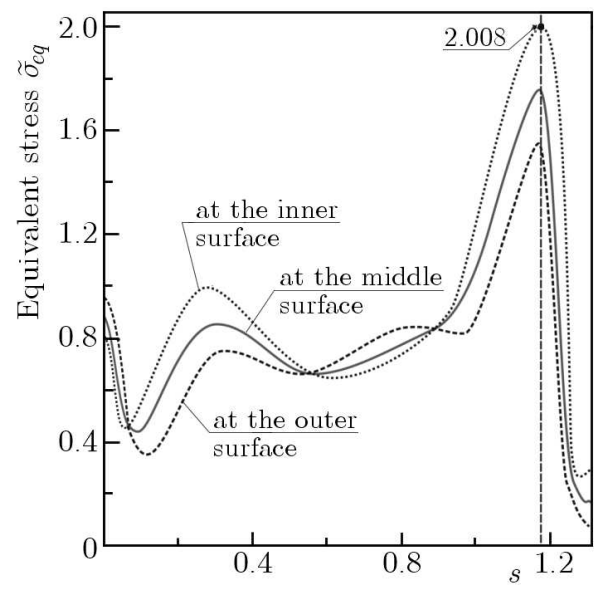

Fig. 2. Equivalent stress at the middle, outer, and inner surfaces of the structure for $e=0$

(axial symmetry)

The coordinate $\mathrm{s}$ is a dimensionless length of the generatrix arc. The value $s=0$ corresponds to the point of connection between the cylindrical part of the vessel and its head. The broken vertical line at the right-hand side of the plot corresponds to the arc length where the nozzle is connected to the head. Further course of $s$ ordinate is equivalent to the straight line generatrix of the nozzle. Unfortunately, the disturbance resulting from the nozzle is conducive to significant growth of the stress above the level of $\sqrt{3} / 2$ in the region where the nozzle connects with the head.

The maximum stress value equal to $\widetilde{\sigma}_{e q}=2.008$ occurs at the internal surface. The stress pattern obtained from the FEM approaches the one found analytically by Magnucki (1998) for a hemispherical head.

Further analysis consists in increasing of value $e$, thus shifting the nozzle from the axis of symmetry of the vessel.

In the case of $e=0.2$, the stress distribution remains relatively similar to that of $e=0$, with the maximum value of the dimensionless equivalent stress growing to 2.162. The plots corresponding to this case are not shown here.

Figure 3 presents the stresses along the upper and bottom generatrices of the head/nozzle system for $e=0.4$. Since the nozzle is now located above the axis of symmetry of the vessel, the interval of variability of the $s$ coordinate is shortened for the upper generatrix and elongated for the bottom one. Comparison with the previous case shows that the stresses changed with their maximum values remaining in the zone of head/nozzle connection. In the case of symmetrical location of the nozzle, the stress along the head/nozzle edge (the arc MN in Fig. 1b marked with broken line) is constant. On the other hand, when $e \neq 0$, it must vary. The effect is shown in Fig. 4. The ordinate $s$ is the dimensionless arc length of the head/nozzle edge, with $s=0$ corresponding to the point $M$ (i.e. intersection with the upper generatrix) and $s=1$ to the point $N$ (intersection with the bottom one). 

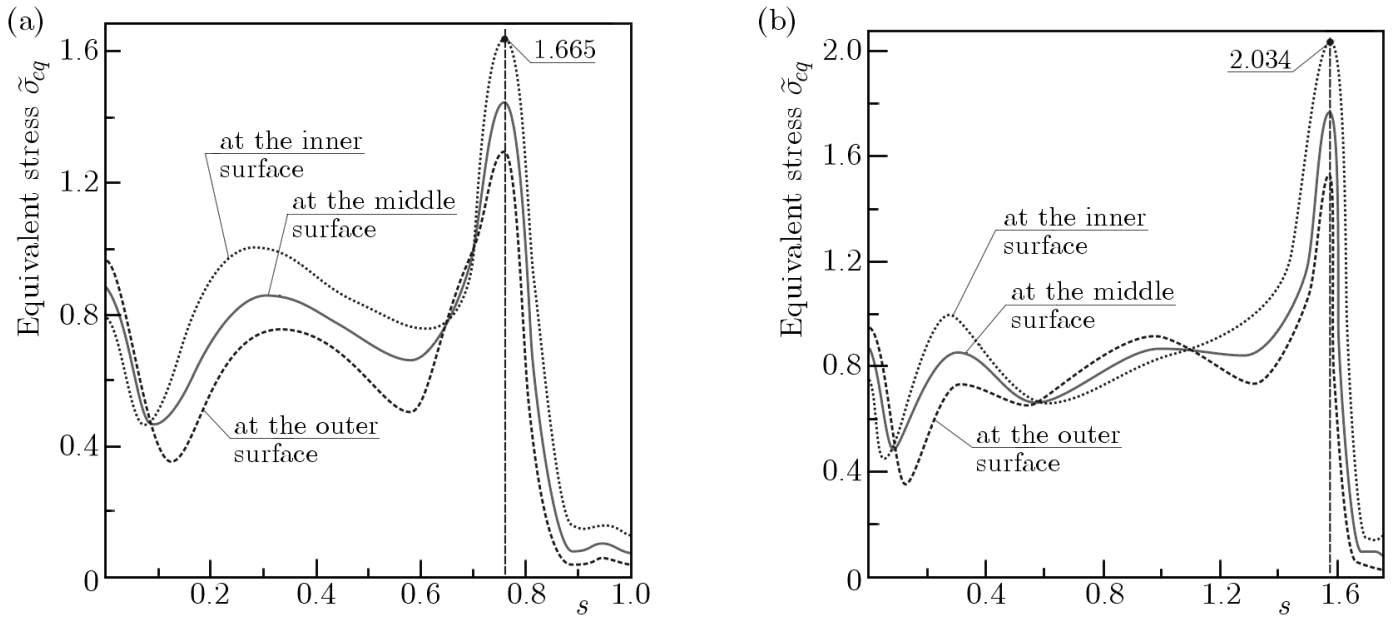

Fig. 3. Equivalent stress at the middle, outer and inner surfaces of the structure for $e=0.4$ : (a) along the upper generatrix, (b) along the bottom generatrix

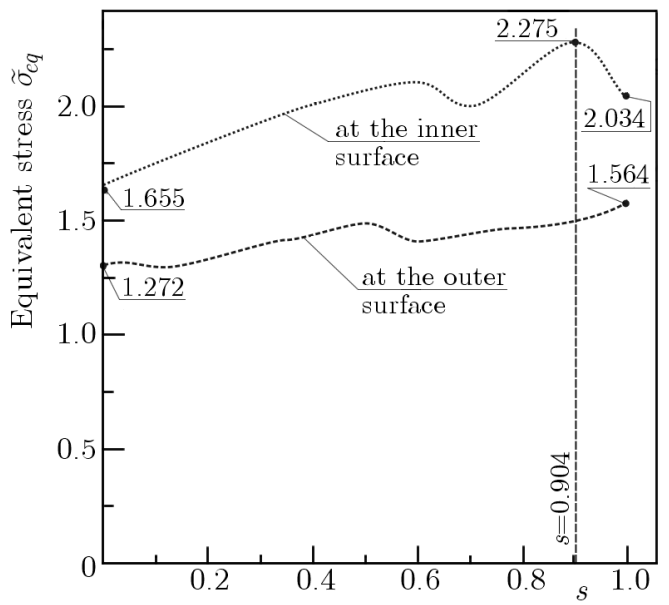

Fig. 4. Equivalent stress at the outer and inner surfaces of the structure along the head/nozzle edge for $e=0.4$

The vertical broken line indicates the point of the maximum stress value. The equivalent stress level oscillates, taking the maximum value of 2.275 at the internal surface for $s=0.904$.

Figure 5 presents an approximate distribution of the dimensionless equivalent stress in the head in the vicinity of the head/nozzle edge at both sides of the shell. The field of the plot is a perpendicular projection of the isolines at the plane perpendicular to the axis of symmetry of the vessel. Therefore, accuracy of the picture is worsened near the upper circular line corresponding to the head/cylinder edge.

Similar calculation has been carried out for the cases $e=0.6$ and $e=0.8$. They are shown in Figs. 6-11. In both cases, the nozzle more and more approximates the upper part of the edge of connection between the head and cylindrical part of the vessel. In consequence, the boundary disturbances generated by the head/nozzle and head/cylindrical part collide with each other.

In the case of $e=0.6$, a large stress concentration is observed at the upper generatrix of the head due to vicinity of both connection zones (Fig. 6a). In the head/nozzle connection zone, the equivalent stress at the upper generatrix is slightly lower. On the other hand, at the bottom generatrix, the stress is more balanced (Fig. 6b) but also exceeds its allowable level $\sqrt{3} / 2$. Maximum stress value occurs approximately in the middle of the head/nozzle edge (Fig. 7), i.e. for $s=0.52$, where it takes the value of $\widetilde{\sigma}_{e q}=2.279$. 

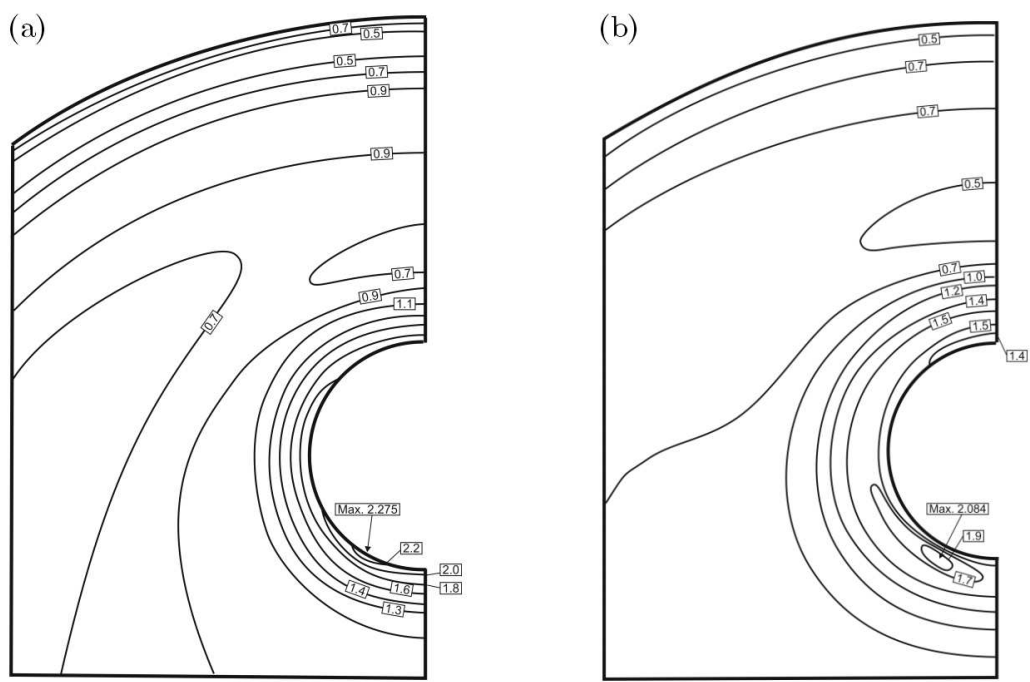

Fig. 5. Equivalent stress distribution in the vicinity of the head/nozzle edge for $e=0.4$ :

(a) at the inner surface, (b) at the outer surface
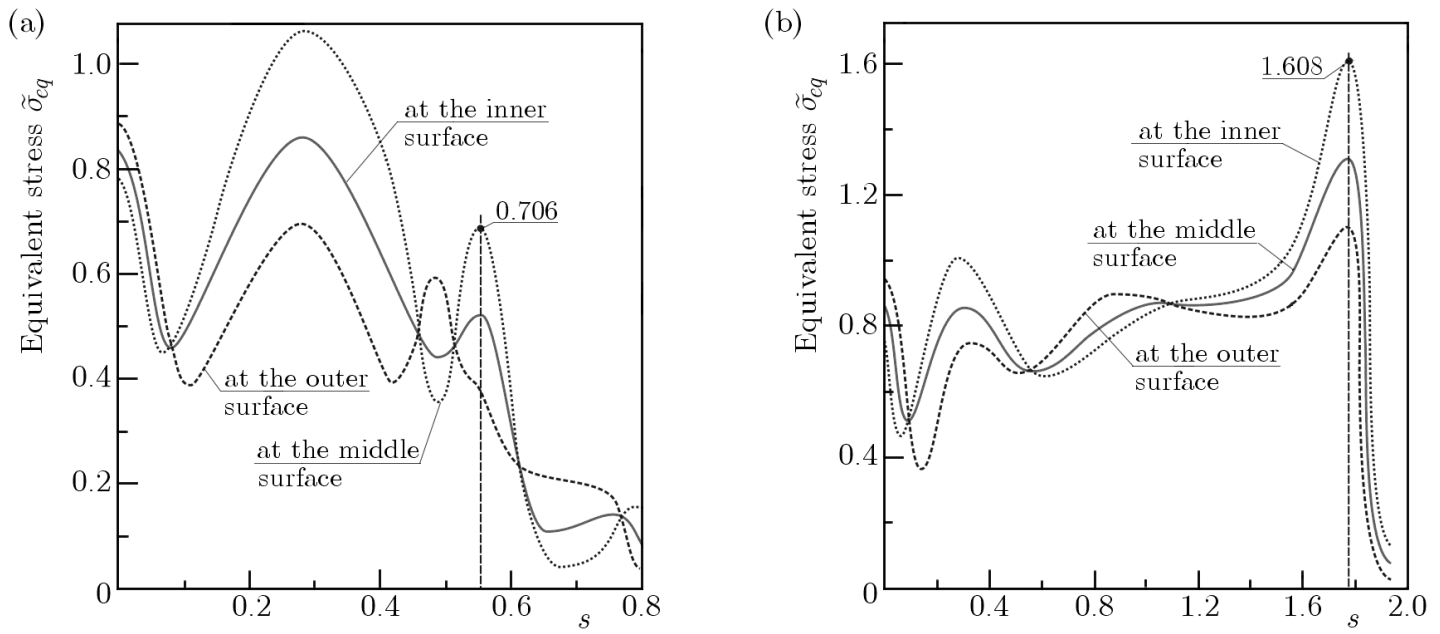

Fig. 6. Equivalent stress at the middle, outer and inner surfaces of the structure for $e=0.6$ :

(a) along the upper generatrix, (b) along the bottom generatrix

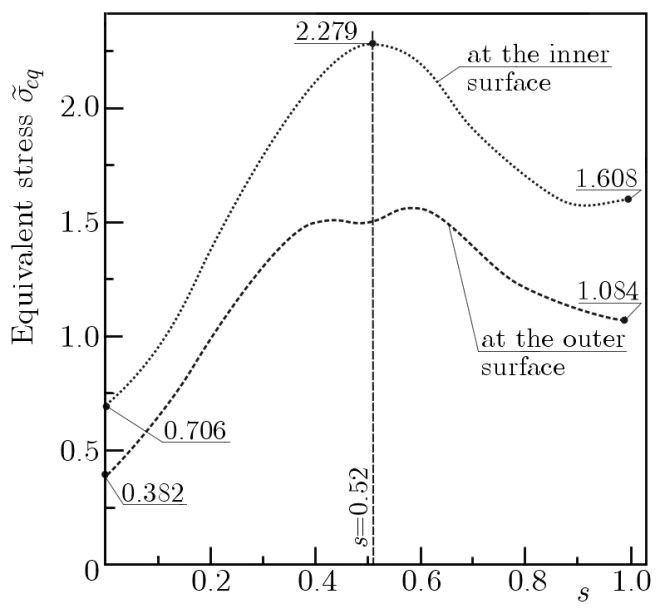

Fig. 7. Equivalent stress at the outer and inner surfaces of the structure along the head/nozzle edge for $e=0.6$ 

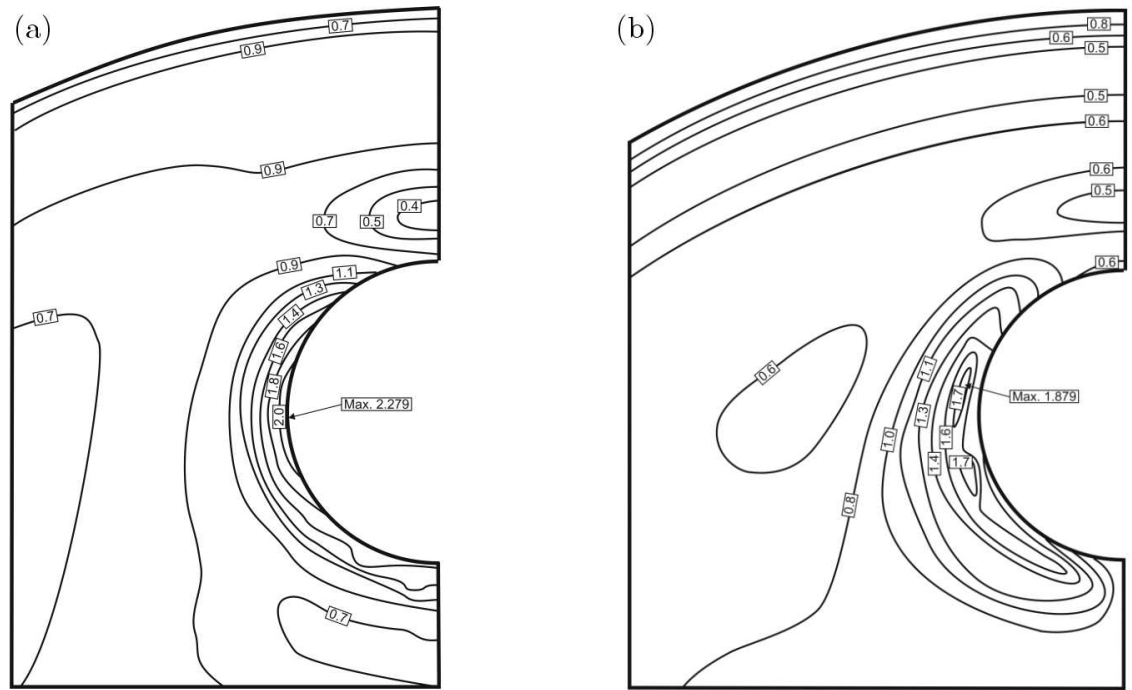

Fig. 8. Equivalent stress distribution in the vicinity of the head/nozzle edge for $e=0.6$ : (a) at the inner surface, (b) at the outer surface
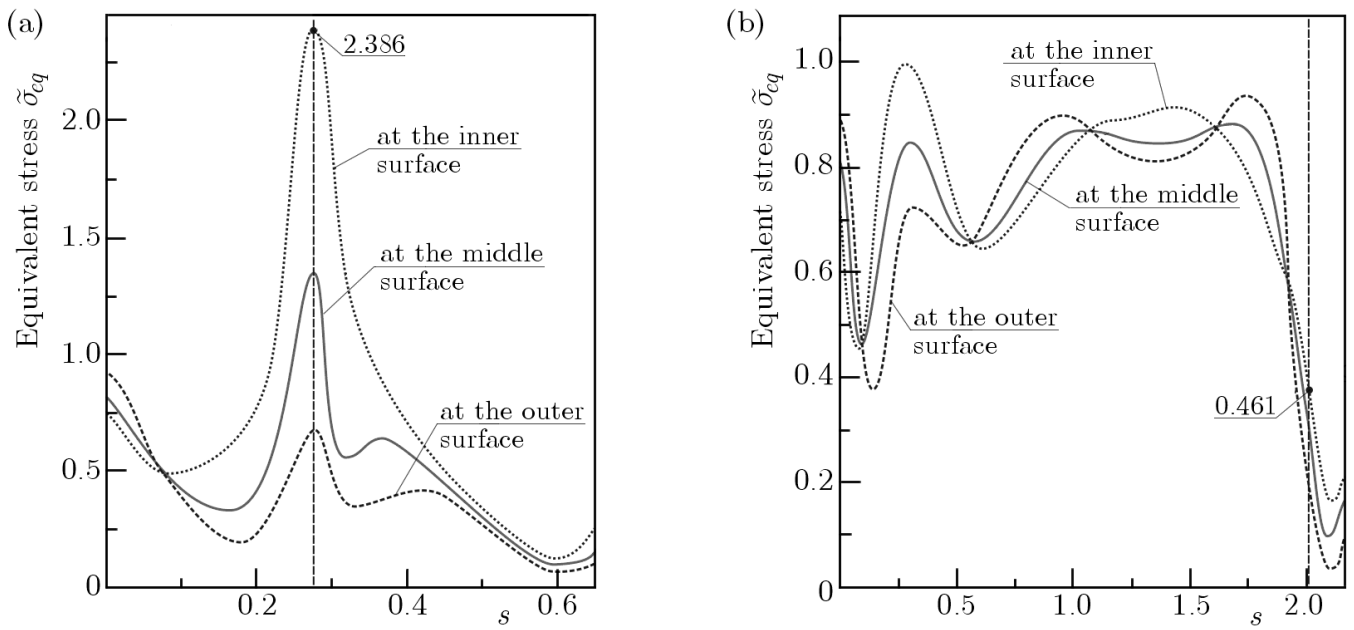

Fig. 9. Equivalent stress at the middle, outer and inner surfaces of the structure for $e=0.8$ :

(a) along the upper generatrix, (b) along the bottom generatrix

Once the nozzle is located at the distance $e=0.8$, the stress pattern changes a little. The maximum equivalent stress on the upper generatrix at the internal shell surface reaches the value $\widetilde{\sigma}_{e q}=2.386$ in the point of head/nozzle connection (Fig. 9a). On the other hand, the stress on the bottom generatrix is lower. Similarly as in the previous case, the maximum stress value $\widetilde{\sigma}_{e q}=2.646$ occurs in the middle of the head-nozzle edge (Fig. 10), for $s=0.49$.

The distribution of the dimensionless equivalent stress in the head in the vicinity of the head/nozzle edge for $e=0.8$ shown in Fig. 11 is rather poorly depicted between the upper part of the head/nozzle edge and the circular line because of the projection of the stress field mentioned already earlier.

It has been found that maximum values of the equivalent stress always occur at the inner shell surface. They significantly exceed the stress level existing in the cylindrical part of the vessel. With growing distance between the axes of symmetry of the nozzle and the vessel (i.e. growing e) the maximum stress grows too.

The plots shown here give a clear evidence of the disadvantageous effect of the nozzle on the head strength. In all the examples presented here, the dimensionless equivalent stress exceeds the level of 2.00 , reaching $\widetilde{\sigma}_{e q}=2.646$ in the most disadvantageous case. 


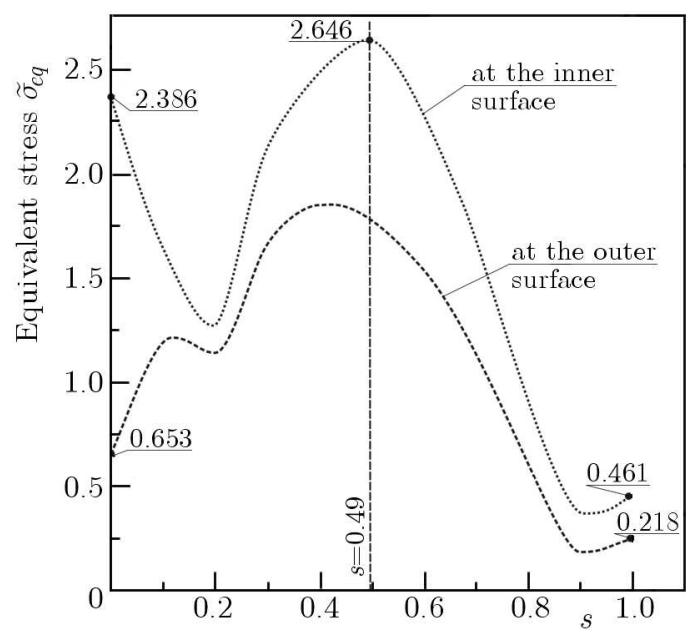

Fig. 10. Equivalent stress at the outer and inner surfaces of the structure along the head/nozzle edge for $e=0.8$
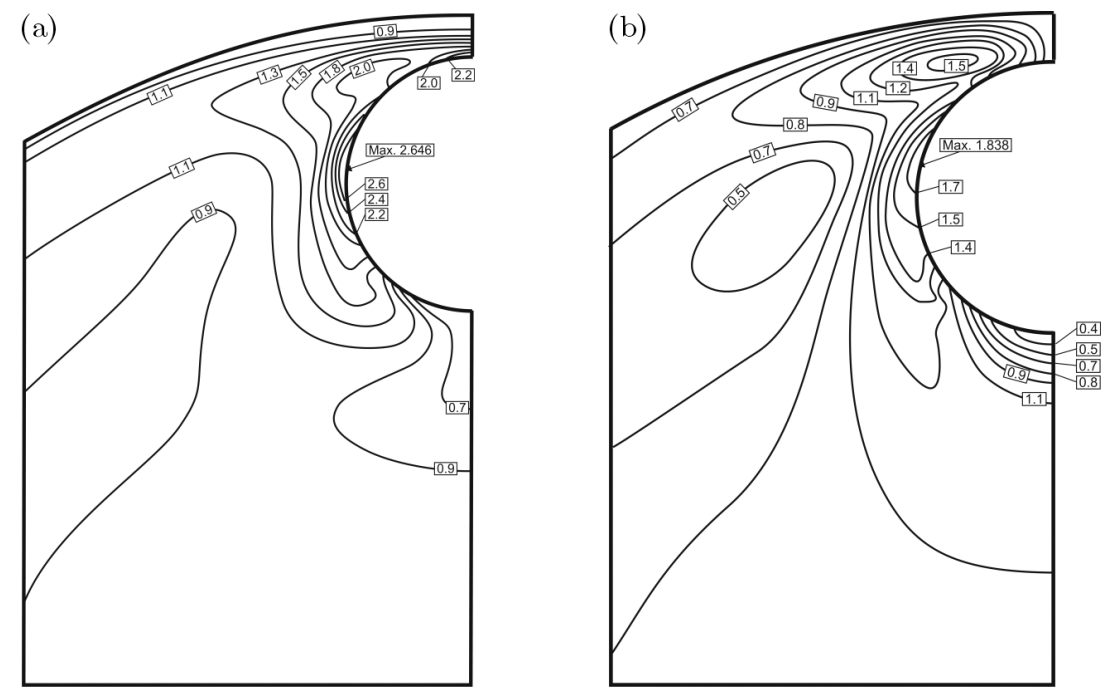

Fig. 11. Equivalent stress distribution in the vicinity of the head/nozzle edge for $e=0.8$ :

(a) at the inner surface, (b) at the outer surface

\section{Proposals aimed at reducing the stress concentration}

In order to reduce the stress down to the value of $\widetilde{\sigma}_{e q}=0.866$ existing in the cylindrical part of the vessel, the head thickness should be locally increased in the head/nozzle zone. This may be achieved by means of a cover plate of a proper thickness.

The stress has been calculated for two variants with cover plates. In both variants, the structure remains a thin-walled one. In consequence, the model is divided into triangular shell elements as before. The first variant consists in modification of the case in which the nozzle is located in the vessel axis of symmetry.

A cover plate in form of a ring of a dimensionless outer diameter equal to $D_{c}=0.5$ has been used, according to the scheme shown in Fig. 12. The dimensionless wall thickness $t$ is equal to 0.01 , while the thickness in the zone reinforced by the cover plate amounts to 0.03 . Therefore, thickness of the shell elements varies accordingly. The plot of the equivalent stress along the generatrix at the inner surface of the shell is shown in Fig. 13.

Comparison with the case presented in Fig. 2, without the cover plate, gives an evidence of significant improvement of the strength. Nevertheless, the equivalent stress exceeds its allowable level and further reinforcement of the structure would be required. 


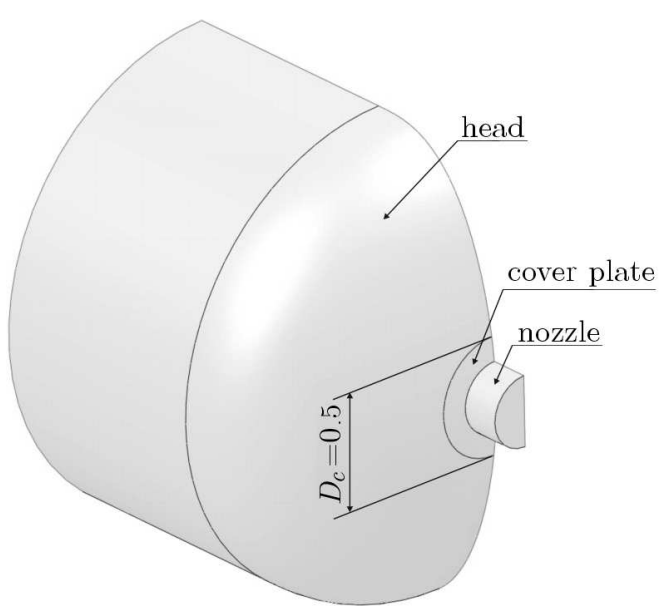

Fig. 12. The head with a symmetrically located nozzle reinforced with a cover plate

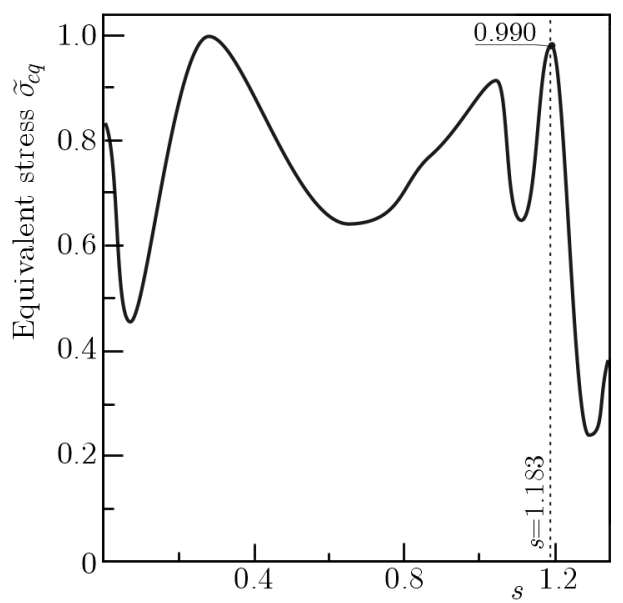

Fig. 13. Equivalent dimensionless stress at the inner surface of the head and nozzle reinforced with a cover plate for $e=0$ (axisymmetric case)

The second variant is an effort of improving the strength of the head/nozzle connection for $e=0.8$, in which the highest stress concentration occurred. A cover plate in form of a generalized ring of a dimensionless outer diameter $D_{c}=0.6$ has been used. The profile of the cover plate results from projection of a ring of this diameter on the head surface. A scheme of such reinforcement is shown in Fig. 14. Similarly as before, the relative wall thickness of the vessel is equal to $t=0.01$, while in the zone reinforced by the cover it grows to 0.03 .

The plot of the equivalent stress along the upper generatrix at the inner surface of the shell is shown in Fig. 15. Comparison with the plot in Fig. 9, where the same nozzle configuration without the cover plate is shown, allows one to expect that such an approach is at least conducive to the required end. The stress is significantly reduced, although it remains too high.

A more sophisticated approach to the problem is presented in Fig. 16a. In this example, only a symmetrical nozzle location is considered. Instead of the cover plate, the head thickness is increased in the area of the nozzle. The outside surface of the head is a part of a sphere, since the arc of dimensionless radius equal to 1.80 is tangent to the previously assumed head shape in the point $T$. The thickness enlarged in the nozzle/head region decreases to its nominal value in a certain distance (here equal to 0.72 ) from the axis of symmetry. Thickness variability is approximately shown in Fig. 16a. The outside and inside edges of the nozzle/head junction are rounded with the dimensionless radius 0.04 . 


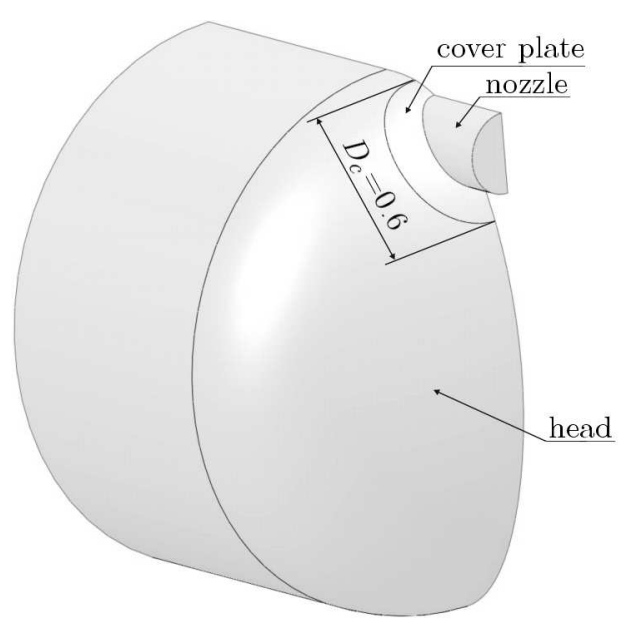

Fig. 14. The head with an eccentrically located nozzle reinforced with a cover plate

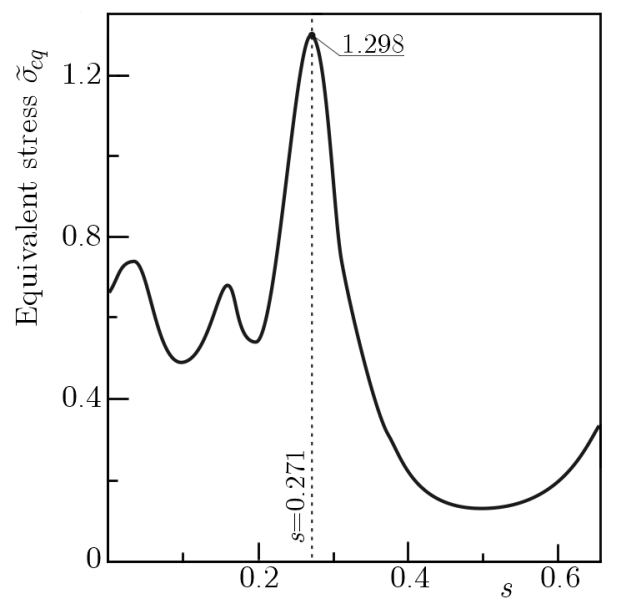

Fig. 15. Equivalent dimensionless stress along the upper generatrix at the inner surface of the head and nozzle reinforced with a cover plate for $e=0.8$

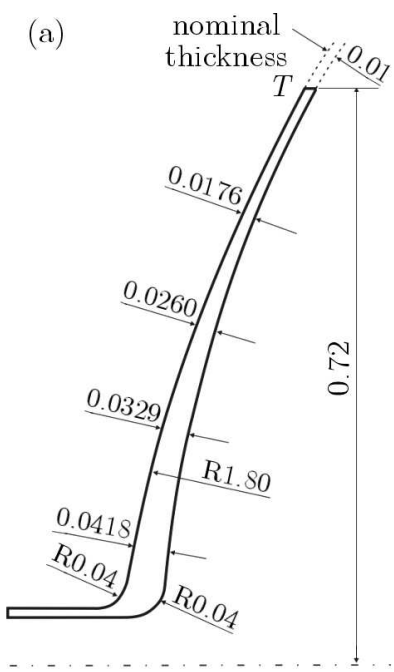

(b)

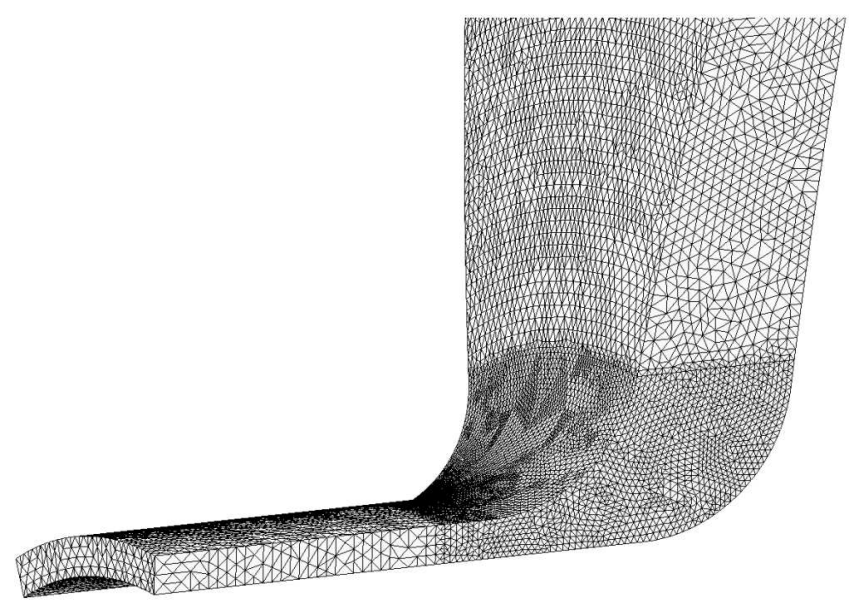

Fig. 16. (a) Proposed shape of the head with gradually varying thickness, (b) exemplary division of a part of the structure into 3D tetrahedral four-node finite elements 
As opposed to earlier cases, where triangular shell elements are used, the model is divided into tetrahedral (four-node) solid elements, as shown in Fig. 16b. The maximum stress value only insignificantly exceeds the reference level $\widetilde{\sigma}_{e q}=0.866$. The distribution of the equivalent dimensionless stress in the cross-section of the nozzle/head region is shown in Fig. 17.

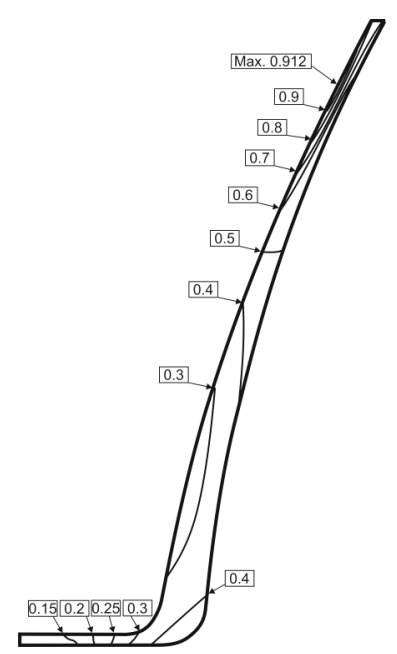

Fig. 17. Equivalent dimensionless stress in the cross-section of the nozzle/head region

Such a solution is better than those with the cover plates. More careful shaping of the part of the head with varying thickness should enable further reduction of the stress. Unfortunately, the technology of production of such a vessel would be much more difficult and presumably more expensive.

\section{Conclusions}

The analysis presented above allows one to find that, without the use of a reinforcing cover plate, the best solution for locating the nozzle in the vessel head is to place it in the axis of symmetry of the vessel or, at least, not far away from it.

The stress concentration arising at the head/nozzle connection edge (at the inner side of the shell) may be alleviated with the use of a cover plate in order to enlarge the thickness of the structure in the connection zone. The dimension and thickness of the cover plate should be found based on FEM calculation related to the geometry of the considered vessel.

Another promising solution consists in shaping the nozzle/head region with gradually varying thickness. In this case, the stress distribution is more advantageous, but manufacturing of such a structure would be probably difficult and expensive.

The present paper considers only the nozzles with the dimensionless diameter $D=0.3$. It is obvious that the stress state for other diameter values would be different. Nonetheless, one could expect that the general character of the stress pattern should be similar.

\section{References}

1. BŁachut J., Magnucki K., 2008, Strength, stability, and optimization of pressure vessels: Review of selected problems, Applied Mechanics Reviews, 61, 6, 1-33

2. Carbonari R.C., Munoz-Rojas P.A., Andrade E.Q., Paulino G.H., Nishimoto K., Solva E.C.N., 2011, Design of pressure vessels using shape optimization: An integrated approach, International Journal of Pressure Vessels and Piping, 88, 198-212 
3. Dekker C.J., Brink H.J., 2000, Nozzles on spheres with outward weld area under internal pressure analysed by FEM and thin shell theory, International Journal of Pressure Vessels and Piping, 77, 399-415

4. Giglio M., 2003, Fatigue analysis of different types of pressure vessel nozzle, International Journal of Pressure Vessels and Piping, 80, 1-8

5. Hsieh M.F., Moffat D.G., Mistry J., 2000, Nozzles in the knuckle region of a torispherical head: limit load interaction under combined pressure and piping loads, International Journal of Pressure Vessels and Piping, 77, 807-815

6. Kruzelecki J., Proszowski R., 2012, Shape optimization of thin-walled pressure vessel end closures, Structural and Multidisciplinary Optimization, 46, 739-754

7. Lewiński J., Magnucki K., 2010, Shaping of a middle surface of a dished head of a circular cylindrical pressure vessel, Journal of Theoretical and Applied Mechanics, 48, 2, 297-307

8. Lewiński J., MAGnuCKI K., 2012, Optimal shaping of middle surface of a dished head of circular cylindrical vessel with the help of Bézier curve, EngOpt 2012 - 3rd International Conference on Engineering Optimization, Rio de Janeiro, Brazil

9. Liu J.S, Parks G.T., Clarkson P.J., 2001, Shape optimization of axisymmetric cylindrical nozzles in spherical pressure vessels subject to stress constraints, International Journal of Pressure Vessels and Piping, 78, 1, 1-9

10. Magnucki K., 1998, The Endurance and the Optimization of Thin-Walled Containers (in Polish), PWN, Warsaw

11. Magnucki K., Lewiński J., 2000, Fully stressed head of a pressure vessel, Thin-Walled Structures, 38, $167-178$

12. Magnucki K., Lewiński J., 2003, Optimal design of an ellipsoidal head of a pressure cylindrical vessel, Proceedings in Applied Mathematics and Mechanics, 3, 517-518

13. Magnucki K., Lewiński J., Stasiewicz P., 2004, Optimal sizes of a ground-based horizontal cylindrical tank under strength and stability constraints, International Journal of Pressure Vessels and Piping, 81, 913-917

14. Magnucki K., Szyc W., Lewiński J., 2002, Minimization of stress concentration factor in cylindrical pressure vessels with ellipsoidal heads, International Journal of Pressure Vessels and Piping, 79, 841-846

15. SaAl H., Bauer H., Hderle M.U., 1997, Flexibility factors for nozzles in the knuckle region of dished pressure vessel heads, International Journal of Pressure Vessels and Piping, 70, 151-160

16. Schindler S., Zeman J.L., 2003, Stress concentration factors of nozzle - sphere connections, International Journal of Pressure Vessels and Piping, 80, 87-95

17. Skopinsky V.N., Smetankin A.B., 2003, Parametric study of reinforcement of pressure vessel head with offset nozzle, International Journal of Pressure Vessels and Piping, 80, 333-343

18. Szybiński B., Wróblewski A., 2011, Parametric optimization of stress relief groove shape in flat ends of boilers, Journal of Strain Analysis, 47, 1, 55-63

19. Ventsel E., Krauthammer T., 2001, Thin Plates and Shells. Theory, Analysis and Applications, Basel: Marcel Dekker, Inc, New York

20. Wittenbeck L., Magnucki K., 2008, Strength shaping of dished heads of pressure cylindrical vessels, Proceedings of the Ninth International Conference on Computational Structures Technology, B.H.V. Topping and M. Papadrakakis (Edit.), Civil-Comp Press, Stirlingshire, Scotland (Paper 138, CD)

21. Zhou S.J., Zhang S.Y., WANG W.G., 1995, Effects of transverse shear on radial flexibility factors of nozzles in pressure vessel heads, International Journal of Pressure Vessels and Piping, 61, 111-116 